\title{
Influencias ejercidas por los álcalis sobre las propiedades del clínker de cemento portland y del cemento portland
}

Existe ya literatura acerca de la incorporación de álcalis en las fases de los clinkeres, así como acerca de la importancia de las fases de clínker que contienen álcalis [1] - [14]. Vamos a informar acerca de los ensayos propios con el fin de completar la literatura ya existente.

Se prepararon en el laboratorio cuatro series de clínkeres que contenían álcalis. A un crudo de cemento sintético sin álcalis compuesto de sustancias químicamente puras se añadieron escalonadamente $\mathrm{K}_{2} \mathrm{SO}_{4}$ (serie $\mathrm{KS}$ ), $\mathrm{Na}_{2} \mathrm{SO}_{4}$ (serie $\mathrm{NS}$ ), $\mathrm{K}_{2} \mathrm{CO}_{3}$ (serie $\mathrm{KC}$ ) y $\mathrm{Na}_{2} \mathrm{CO}_{3}$ (serie NC). En los clínkeres se incorporó de 0 a $3 \% \mathrm{~K}_{2} \mathrm{O}$ ó 0 a $4 \% \mathrm{Na}_{2} \mathrm{O}$ (designación de las muestras: por ejemplo $0,8 \mathrm{KC}$ significa $0,8 \% \mathrm{~K}_{2} \mathrm{O}$ en el clínker por adición de $\mathrm{K}_{2} \mathrm{CO}_{3}$ al crudo). Junto a estos clínkeres que continen álcalis se investigó una muestra sin álcalis (muestra 0). Todos los clínkeres presentaron un standard de cal de 91 , un módulo de silicatos de 1,6 y un módulo de fundentes de 2,2.

Los clínkeres fueron investigados químicamente, microscópicamente y radiográficamente. De todos ellos se prepararon cementos de la misma finura y del mismo contenido de $\mathrm{SO}_{3}$. En todos los cementos se mantuvo constante el contenido total de $\mathrm{SO}_{3}$ (clínker- $\mathrm{SO}_{3}$ y yeso- $\mathrm{SO}_{3}$ ). En estos cementos se determinó la resistencia a la compresión, la resistencia a la temperatura ambiente, el comportamiento en el fraguado y el grado de hidratación.

\section{Influencia de los carbonatos alcalinos en las fases de los clínkeres}

Hay que considerar aquí las cuatro fases: alita, belita, aluminato cálcico y ferritoaluminato cálcico.

En los campos investigados se incorporaron totalmente los álcalis en las fases del clínker. La proporción predominante se encuentra siempre en la fracción aluminato-ferrito. Esta fracción tiene una concentración de $\mathrm{K}_{2} \mathrm{O} 3$ a 5 veces superior, o una concentración de $\mathrm{Na}_{2} \mathrm{O} 4$ a 10 veces superior, que la de los silicatos. Especialmente está claro, en el caso de concentraciones reducidas de álcalis, el enriquecimiento relativamente fuerte en la fracción aluminato-ferrito. Esto muestra que, especialmente con una baja aportación de álcalis, éstos se incorporan preferentemente en el aluminato cálcico. Entre los silicatos 
alita y belita, la belita puede incorporar claramente más álcalis que la alita. Esto resulta claro por el hecho de que con un contenido total de silicatos constante, pero con una relación alita/belita de 5:2, los silicatos incorporaron sólo de 25 a $30 \%$ de la cantidad aportada de $\mathrm{K}_{2} \mathrm{O}$; por el contrario, con una relación de 1:1 incorporaron $40 \%$ aproximadamente. La misma regla se puede aplicar a la incorporación de $\mathrm{Na}_{2} \mathrm{O}$.

\section{Alita}

La alita presenta, al crecer el contenido en álcalis del clínker, tamaños crecientes de cristal. Existe en todos los clínkeres, en la modificación monoclínica $\mathrm{M}_{\mathrm{I}}$ de la red $\mathrm{Ca}_{3} \mathrm{SiO}_{5}$ según Woermann, Hahn y Eysel [15]. Por la incorporación de los álcalis no se influye sobre la construcción de la red, ya que por la incorporación de $\mathrm{Al}_{2} \mathrm{O}_{3}$ y $\mathrm{MgO}$, incluso en la muestra 0 carente de álcalis, está estabilizada la modificación monoclínica $\mathrm{M}_{\mathrm{I}}$. El $\mathrm{Na}_{2} \mathrm{O}$ está incorporado con toda seguridad en la alita de las muestras investigadas, lo que demuestra el aumento cuantitativo de alita con contenidos relativamente reducidos de $\mathrm{Na}_{2} \mathrm{O}$.

\section{Belita}

Al contemplar con el microscopio de luz reflejada la belita de la muestra 0 aparece de un color claro homogéneo; puede apreciarse una débil laminación. Las belitas de las series de los carbonatos de sodio y de potasio, al crecer el contenido de álcalis, muestran una reducción de láminas que se reflejan claramente y, con ello, un aumento de campos no laminados que reflejan un matiz obscuro (fig. 1).

En un examen radiográfico la belita de la muestra 0 se puede identificar claramente y de manera predominante como modificación $\beta-\mathrm{C}_{2} \mathrm{~S}$. En la serie de carbonato potásico, con creciente contenido de $\mathrm{K}_{2} \mathrm{O}$, existen a la vez en forma creciente las modificaciones $\alpha$ y $\alpha$ ' La muestra con $0,4 \%$ de $\mathrm{K}_{2} \mathrm{O}$ contiene preponderantemente $\beta-\mathrm{C}_{2} \mathrm{~S}$. La muestra con $3 \%$ de $\mathrm{K}_{2} \mathrm{O}$ es predominante la modificación $\alpha-\mathrm{C}_{2} \mathrm{~S}$ (fig. 2). En la serie de carbonato sódico es clara la misma tendencia con una intensidad aproximadamente igual.

De este modo la investigación demuestra que en la belita se incorporan tanto el sodio como el potasio.

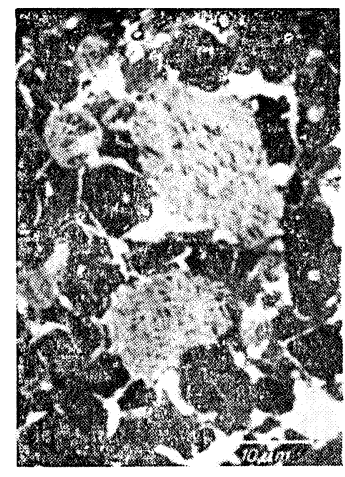

Sin álcalis

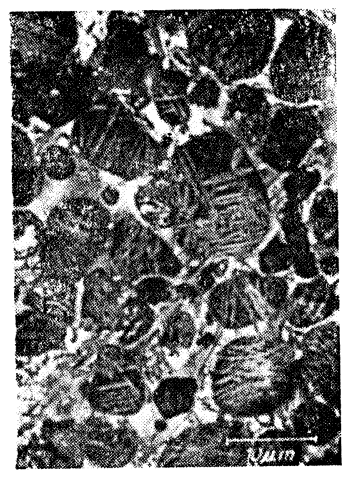

Con álcalis

Fig. 1.-Corte de un clínker: Belita (fases redondeadas y laminares).

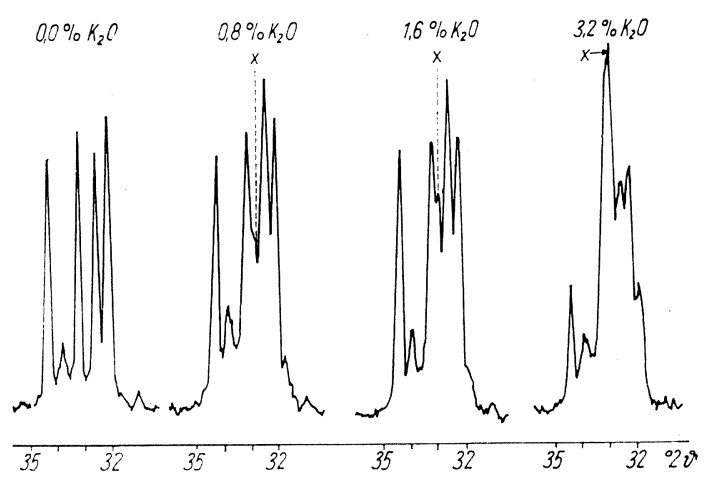

Fig. 2.-Incremento de $\alpha-\mathrm{C}_{2} \mathrm{~S}$ (serie $\mathrm{KC}$ ). 
Teniendo en cuenta los resultados radiográficos es probable que en los campos que se reflejan obscuros se trate de modificaciones $\alpha-\mathrm{C}_{2} \mathrm{~S}$, y en las láminas claras se trate de $\alpha$ ' o $\beta-C_{2} S$. Probablemente las láminas claras estén a su vez laminadas, si se trata de $\beta-C_{2} S$, de manera que exista aquí $\beta$-paramorfosis según las láminas $\alpha$ '.

Algo análogo pudo comprobar Trojer [16] con el microscopio electrónico.

La belita de la muestra 0 está lobulada, es decir, las láminas después de su transformación de $\alpha \rightarrow \alpha^{\prime} \mathrm{C}_{2} \mathrm{~S}$ han crecido más, por encima de la forma redondeada original del $\alpha-\mathrm{C}_{2} \mathrm{~S}$. Las muestras ricas en álcalis no muestran esta apariencia, pues en ellas, mediante la incorporación de álcalis, se ha desplazado el punto de transformación $\alpha \rightarrow \alpha$ ' a temperaturas inferiores. A estas temperaturas ya no era posible otro crecimiento de cristal (fig. 3).

\section{Aluminato cálcico}

El aluminato cálcico $\left(\mathrm{C}_{3} \mathrm{~A}\right)$ es xenomorfo en la muestra 0 y aparece en el ensayo con una superficie irregular.

En la serie de carbonato potásico, en las muestras pobres en álcalis, los aluminatos son, en su mayor parte, igualmente xenomorfos. Con el contenido creciente en álcalis aumentan los cristales idiomorfos, en forma de regletas, y en una medida aproximadamente igual decrecen los cristales xenomorfos. Sólo el aluminato de la muestra más rica en álcalis con un $3 \%$ de $\mathrm{K}_{2} \mathrm{O}$ aparece totalmente en forma de regleta (fig. 4).

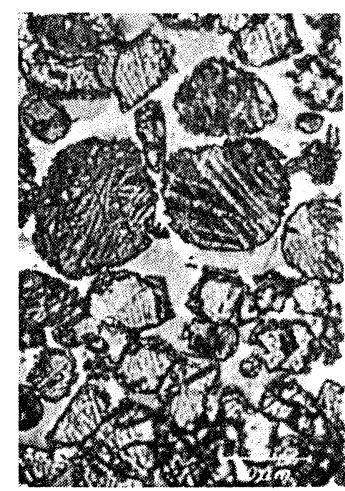

Sin álcalis

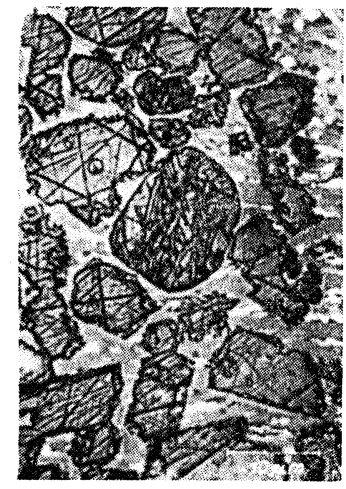

Con álcalis

Fig. 3.-Corte de un clínker: Belita (fases redondeadas y laminares).

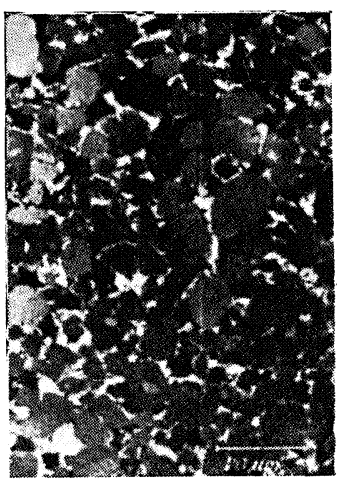

Sin álcalis

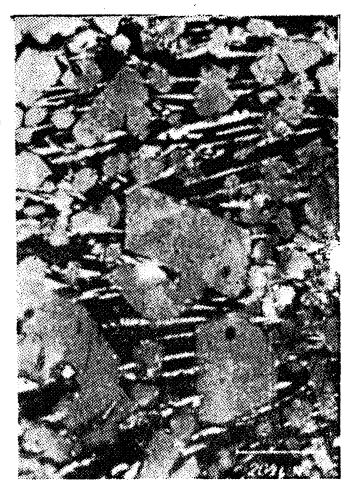

Con álcalis

Fig. 4.-Corte de un clínker: aluminato cálcico (negro).

En la serie de carbonato sódico, el aluminato cálcico es, ya en la muestra más pobre de álcalis con $0,6 \%$ de $\mathrm{Na}_{2} \mathrm{O}$, exclusivamente idiomorfo, en forma de regleta. Esta es la forma típica de cristal para el "aluminato cálcico-alcalino".

Tal como podía esperarse y de manera completamente paralela a ésta las radiografías dan como resultado que en el caso de los cristales xenomorfos existe $\mathrm{C}_{3} \mathrm{~A}$ y en el caso de los cristales idiomorfos se da $\mathrm{NC}_{3} \mathrm{~A}_{3}$ ó $\mathrm{KC}_{8} \mathrm{~A}_{3}$ (fig. 5).

A igualdad de concentraciones en el clínker, el $\mathrm{Na}_{2} \mathrm{O}$ tiene una mayor influencia sobre el aluminato de calcio que el $\mathrm{K}_{2} \mathrm{O}$. 


\section{Aluminoferrito de calcio}

El ferrito aparece en la imagen microscópica de la misma manera en todas las muestıas. Asimismo, por difractometria, sólo en la serie del carbonato sódico se puede comprobar una modificación sistemática.

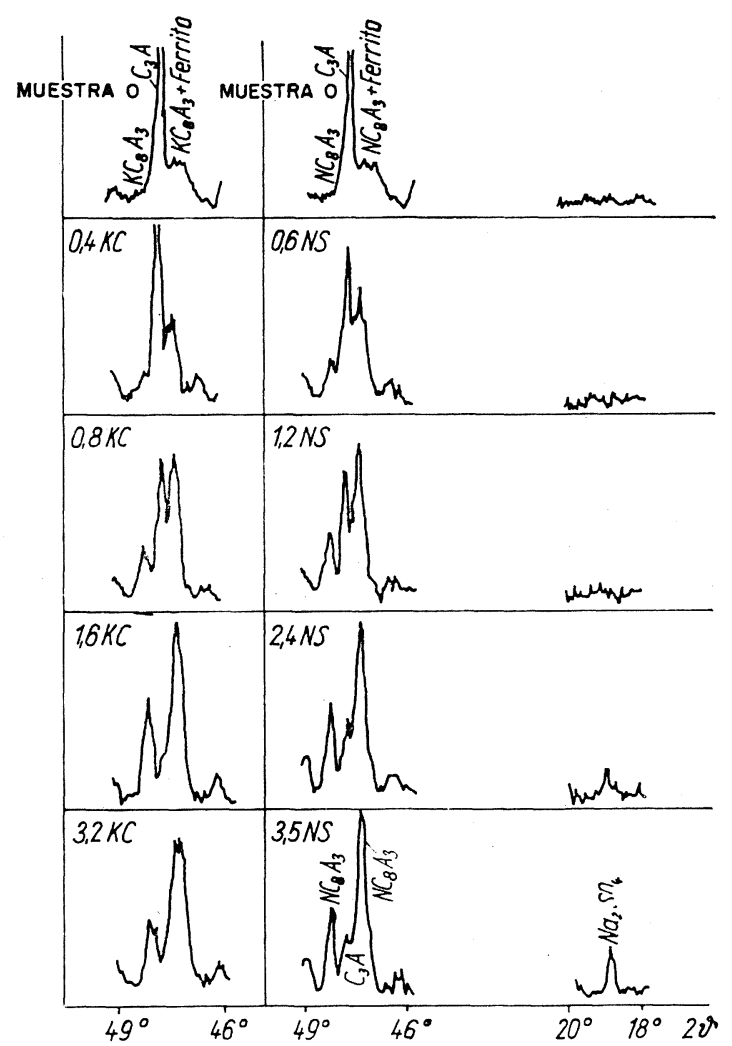

Fig. 5.-Análisiss roentgenográfico de clínker (sin silicatos). Alteración de los aluminatos.

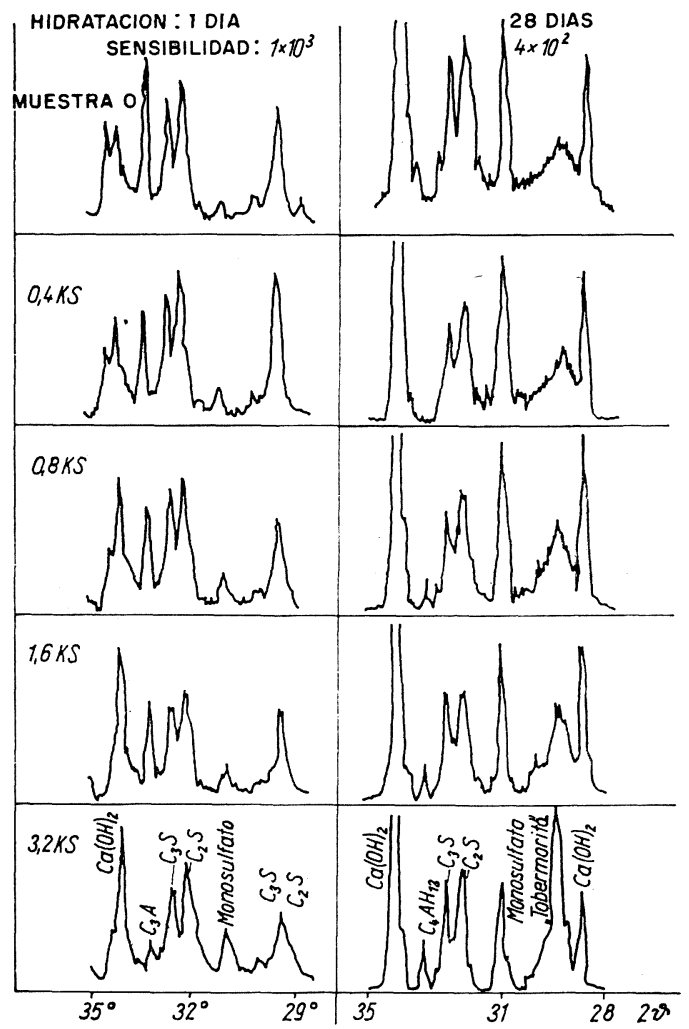

Fig. 6.-Análisis roentgenográfico de cementos hidratados (serie KS).

El contenido en $\mathrm{Fe}_{2} \mathrm{O}_{3}$ del aluminoferrito de calcio aumenta en un $10 \%$ aproximadamente con el contenido creciente de $\mathrm{Na}_{2} \mathrm{O}$ del clínker; en el mismo orden descienden los contenidos de $\mathrm{Al}_{2} \mathrm{O}_{3}$ y CaO. Un trabajo de Smolczyk [17] constituye la base de esta evaluación.

Es condición previa de esta indicación que no fue la incorporación de $\mathrm{Na}_{2} \mathrm{O}$ la que modificó la red cristalina de la fase ferrito. No obstante, es probable que el contenido de $\mathrm{Fe}_{2} \mathrm{O}_{3}$ de esta fase se elevase, pues, simultáneamente, el contenido de aluminato aumentó y el contenido de ferrito disminuyó.

Por consiguiente, no pudo comprobarse la incorporación de álcalis en la fase ferrítica.

\section{Influencia de los sulfatos alcalinos sobre las fases del clínker}

Los clínkeres que contienen sulfatos alcalinos contienen óxidos alcalinos y $\mathrm{SO}_{3}$ estequiométricamente en la relación de 1:1. Esto indica que los sulfatos alcalinos se encuentran en el clínker como fases estables, al menos en lo esencial. 
El sulfato potásico no mostró ni en la formación de las fases ni en la distribución de las mismas influencia clara. Se pudo comprobar claramente el sulfato potásico en el clínker. De este modo se confirmaron los resultados de Taylor [1]: el $\mathrm{K}_{2} \mathrm{SO}_{4}$ es estable en presencia de las fases del clínker. El sulfato sódico, por el contrario, influye en la formación de las fases alita, belita y aluminato con la misma tendencia que el carbonato sódico, aun cuando de manera decididamente menor, pues a la temperatura de sinterización sólo se puede disponer de una fracción del óxido de sodio para la formación de las fases del clínker. Los clínkeres con un contenido creciente de sulfato sódico presentan, junto al $\mathrm{C}_{3} \mathrm{~A}$, aluminatos idiomorfos en forma de regletas, que se pueden identificar por difractometría como $\mathrm{NC}_{8} \mathrm{~A}_{3}$. Ya con $0,6 \%$ de $\mathrm{Na}_{2} \mathrm{O}$ se puede reconocer el $\mathrm{NC}_{8} \mathrm{~A}_{3}$, pero, por otra parte, se puede comprobar que con $4,8 \%$ de $\mathrm{Na}_{2} \mathrm{O}$ también existe $\mathrm{C}_{3} \mathrm{~A}$ junto a $\mathrm{NC}_{8} \mathrm{~A}_{3}$. En la belita, con el contenido creciente de $\mathrm{Na}_{2} \mathrm{O}$, se estabilizan de manera cada vez mayor las modificaciones $\alpha \mathrm{y} \alpha^{\prime}$ del $\mathrm{C}_{2} \mathrm{~S}$.

No se ha podido reconocer una modificación del aluminoferrito de calcio. Por eso no se han podido confirmar plenamente los resultados que Eubank [2]. Krämer y Zur Strassen [18] encontraron, en clínkeres que contenían $2 \%$ de $\mathrm{Na}_{2} \mathrm{SO}_{4}$ : belita junto a cal libre en forma estable y además una formación de alita esencialmente reducida. No se ha podido confirmar esta observación en este trabajo dentro de la serie NS, pero sí en la serie NC.

\section{Influencia de los álcalis en la distribución cuantitativa de fases}

En la serie de carbonato sódico aumenta al principio ligeramente el contenido de alita, y simultáneamente desciende ligeramente el contenido de belita. La causa de esto podría ser la incorporación de $\mathrm{Na}_{2} \mathrm{O}$ en la alita. En los contenidos de $\mathrm{Na}_{2} \mathrm{O}$ superiores al 1,2 \% desciende por el contrario rápidamente el contenido de alita al mismo tiempo que aumenta el contenido de belita y cal libre. La causa de esto es que la belita rica en álcalis junto con la cal libre es estable a la temperatura de sinterización y con ello reduce o impide la formación de alita. El contenido de aluminato cálcico aumenta continuamente por la incorporación de $\mathrm{Na}_{2} \mathrm{O}$. Por el contrario, desciende el contenido de ferrito, al disminuir el $\mathrm{Al}_{2} \mathrm{O}_{3}$ disponible.

En la serie $\mathrm{KC}$ se ve aproximadamente la misma tendencia, pero más débil.

Los sulfatos alcalinos no modifican el contenido cuantitativo de las fases en forma práctica.

En [19] se encuentran los valores numéricos y las figuras a este respecto.

\section{Influencia de los álcalis en la hidratación}

La figura 6 muestra gráficamente un ejemplo de las tendencias observadas en los productos de hidratación así como en las fases de los clínkeres haciendo uso de là difracción de rayos X. Para esto se ha elegido la serie de sulfato potásico, pues estos clínkeres tienen en conjunto la misma composición mineral.

Si se comparan las muestras tras un día de hidratación se puede observar que con un contenido creciente de álcalis, se hacen menores los picos de aluminato cálcico y silicato cálcico; por el contrario, el $\mathrm{Ca}(\mathrm{OH})_{2}$ y el monosulfato aumentan claramente. Por consiguiente, la hidratación se acelera con contenidos crecientes de álcalis. 
Si se comparan las muestras después de 28 días de hidratación el pico del silicato cálcico hidratado aumenta con el contenido creciente de álcalis; por el contrario, se reduce el pico de $\mathrm{Ca}(\mathrm{OH})_{2}$. Esto significa que con el creciente contenido de álcalis o bien aumenta el contenido absoluto de los silicatos cálcicos hidratados, o bien aparece una forma de CSH mejor cristalizada o posiblemente se forman hidratos de silicatos más ricos en cal, que suministran interferencias más claras. Según Dosch aparece un CSH mejor cristalizado.

Pero había que averiguar claramente que, con una adición creciente de álcalis, se presenta una desintegración acelerada de la ettringita con una formación creciente de monosulfato.

\section{TAB L A 1}

Resistencia a compresión en $\mathrm{kp} / \mathrm{cm}^{2}$ (controlado sobre cilindros de clínker).

\begin{tabular}{clllll}
\hline Serie KS & $\mathbf{0 , 0}$ & $\mathbf{0 , 4}$ & $\mathbf{0 , 8}$ & $\mathbf{1 , 6}$ & $\mathbf{3 , 2}$ \\
\hline 1 DIA & 252 & 262 & 274 & 249 & 204 \\
3 DIAS & 276 & 284 & 276 & 261 & 251 \\
7 DIAS & 315 & 313 & 316 & 302 & 263 \\
28 DIAS & 398 & 423 & 436 & 381 & 339 \\
\hline Serie KC & $\mathbf{0 , 0}$ & $\mathbf{0 , 4}$ & $\mathbf{0 , 8}$ & $\mathbf{1 , 6}$ & $\mathbf{3 , 2}$ \\
\hline 1 DIA & 252 & 249 & 265 & 268 & 161 \\
3 DIAS & 276 & 285 & 290 & 294 & 175 \\
7 DIAS & 315 & 304 & 324 & 297 & 207 \\
28 DIAS & 398 & 421 & 435 & 406 & 272 \\
\hline Serie NS & $\mathbf{0 , 0}$ & $\mathbf{0 , 6}$ & $\mathbf{1 , 2}$ & $\mathbf{2 , 4}$ & $\mathbf{3 , 5}$ \\
\hline 1 DIA & 252 & 252 & 251 & 196 & 140 \\
3 DIAS & 276 & 277 & 264 & 230 & 183 \\
7 DIAS & 315 & 314 & 287 & 251 & 193 \\
28 DIAS & 398 & 445 & 394 & 310 & 242 \\
\hline Serie NC & $\mathbf{0 , 0}$ & $\mathbf{0 , 6}$ & $\mathbf{1 , 2}$ & $\mathbf{2 , 4}$ & $\mathbf{4 , 8}$ \\
\hline I DIA & 252 & 253 & 265 & 137 & 89 \\
3 DIAS & 276 & 253 & 273 & 156 & 68 \\
7 DIAS & 315 & 297 & 309 & 159 & 70 \\
28 DIAS & 398 & 393 & 387 & 227 & 89 \\
\hline
\end{tabular}

La determinación del agua químicamente combinada suministró una indicación adicional de la hidratación inicialmente acelerada por medio de álcalis. Se determinó esta por medio de un calentamiento de una hora a $550^{\circ} \mathrm{C}$ después de un tratamiento previo de la pasta de cemento hidratada ( 24 horas en acetona, vuelta a lavar con éter, calentamiento al vacío durante 2 horas a $40^{\circ} \mathrm{C}$ ).

$\mathrm{El}$ agua combinada químicamente en las muestras que contenían $\mathrm{K}_{2} \mathrm{O}$ y $\mathrm{Na}_{2} \mathrm{O}$ permanece en general, en los términos anteriores de examen $(1,3,7$ d) más alta que en la muestra sin álcalis. Pero, con el tiempo, el aumento es menor, de forma que ya después de 28 días, generalmente, el agua combinada químicamente de la muestra sin álcalis es superior (fig. 7). 
El principio de fraguado, determinado por medio de las curvas tiempo-temperatura ( 40 $\mathrm{g}$ cemento; $\mathrm{w}=0,5$; en cubeta termostata), se reduce esencialmente con un contenido creciente de álcalis. Esta tendencia es más marcada en los clínkeres sin sulfato que en los que lo contienen. Como ejemplo de la figura 8 muestra las curvas tiempo-temperatura de la serie $\mathrm{KC} \mathrm{y} \mathrm{KS}$. La fuerte subida de la temperatura es una relativa medida del principio de fraguado.

La resistencia a la compresión se determinó en pequeños cilindros $(1 \mathrm{~cm} \varnothing, 1 \mathrm{~cm}$ altura, cemento: arena $1: 3$, arena de tres tamaños) después de conservados en agua. Los contenidos reducidos de álcalis no tienen influencia alguna sobre el desarrollo de la resistencia (tabla 1). La figura 9 muestra como ejemplo la influencia de $\mathrm{K}_{2} \mathrm{O}$. Sólo con contenidos de $\mathrm{K}_{2} \mathrm{O}$ superiores a $1,6 \%$ se puede comprobar una clara disminución de las resistencias. De manera más fuertemente desfavorable se influyó sobre las resistencias en plazos de ensayo más avanzados, especialmente después de 90 días. La influencia del $\mathrm{Na}_{2} \mathrm{O}$ corresponde aproximadamente a la del $\mathrm{K}_{2} \mathrm{O}$; sin embargo, aparece ya una clara disminución de resistencia en los contenidos de $\mathrm{Na}_{2} \mathrm{O}$ superiores al $1,2 \%$.

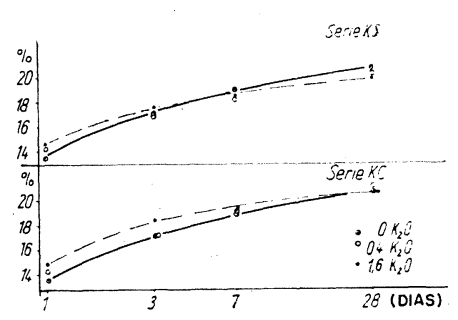

Fig. 7.-Agua ligada quimicamente.

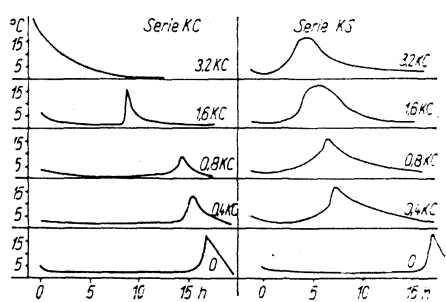

Fig. 8.-Curvas temperaturatiempo.

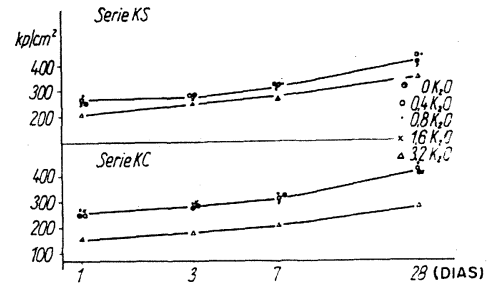

Fig. 9.-Resistencia a compresión (controlado sobre cilindros de clínker).

No se observó una influencia directa de los álcalis sobre la consistencia espacial. Pero allí donde - condicionadas por elevados contenidos ae álcalis $(3,2 \mathrm{KC} ; 4,8 \mathrm{NC})$ - se presentan notables cantidades de cal libre, no se dio la consistencia espacial tal como era de esperar. Si en los clínkeres o cementos, respectivamente, se dan $\mathrm{K}_{2} \mathrm{O}$ y $\mathrm{Na}_{2} \mathrm{O}$, entonces sus influencias son aditivas.

\section{R E S U M E N}

Casi todos los crudos de cemento contienen álcalis, que en parte se incorporan a los clínkeres de cemento portland y pueden modificar sus propiedades.

Se prepararon y examinaron cuatro series de clínkeres, conteniendo álcalis, compuestos de substancias químicamente puras. Se distinguen de la muestra 0 carente de álcalis (standard de cal 91 ; módulo de silicatos 1,6; módulo de fundentes 2,2) sólo por las adiciones de álcalis $\left(\mathrm{K}_{2} \mathrm{SO}_{4}, \mathrm{~K}_{2} \mathrm{CO}_{3}, \mathrm{Na}_{2} \mathrm{SO}_{4}, \mathrm{Na}_{2} \mathrm{CO}_{3}\right.$ hasta $4 \%$ de óxido alcalino en el clínker) al crudo.

Los clínkeres que contienen sulfatos, contienen óxidos alcalinos y $\mathrm{SO}_{3}$ estequiométricamente en relación de $1: 1$. Con una aportación creciente los álcalis se incorporan en forma creciente a los silicatos, pero nada de $\mathrm{SO}_{3}$ prácticamente. La fracción aluminato cálcicoferroaluminato cálcico contiene la parte mayor de álcalis. 
Con la adición creciente de álcalis aumenta el tamaño de cristalización de los silicatos. El $\mathrm{Na}_{2} \mathrm{SO}_{4}$ influye adicionalmente en la composición y formación del aluminato cálcico (en parte formación de "aluminato cálcico alcalino"). Los carbonatos alcalinos permiten igualmente la formación de "aluminato cálcico alcalino" y estabilizan además las modificaciones de belita de simetría superior que contiene álcalis $\left(\alpha\right.$ y $\left.\alpha^{\prime}-\mathrm{C}_{2} \mathrm{~S}\right)$. En el caso de contenidos elevados de óxidos alcalinos en el clínker, que no están combinados con el sulfato, la "belita alcalina" (la solución firme de álcalis en la belita) impide la formación de alita. Esta belita se da, junto a la cal libre, como fase estable a la temperatura de sinterización.

La distribución cuantitativa de fases se modifica decisivamente debido a las elevadas adiciones de carbonatos alcalinos (especialmente: reducción del contenido de alita y aumento del contenido de belita).

De los clínkeres se prepararon cementos de la misma finura y del mismo contenido total de $\mathrm{SO}_{3}\left(\mathrm{SO}_{3}\right.$ del clínker $+\mathrm{SO}_{3}$ del yeso $)$.

Las resistencias a la compresión de los cementos con contenidos reducidos de álcalis (de acuerdo con la práctica) no muestran una modificación clara en relación con la muestra carente de álcalis. Cementos de elevados contenidos de álcalis desarrollan, no obstante, de manera clara resistencias inferiores y presentan un desarrollo más suave en lo que se refiere a los aumentos de resistencia. El comienzo del fraguado se acorta con el contenido creciente de álcalis, pero los álcalis no influyen directamente en la consistencia espacial.

Las muestras que contienen alcalino presentan más agua químicamente combinada (hidratación reforzada) que la muestra carente de álcalis y esto en las primeras edades de la hidratación. Pero en el caso de las muestras que contienen álcalis el aumento es menor con el tiempo.

Coincidiendo con esto la difracción de rayos $\mathrm{X}$ exhibe una hidratación reforzada de las muestras ricas en álcalis. Con una adición creciente de álcalis se puede observar una menor formación de ettringita y una mayor formación de aluminato monosulfato.

$\mathrm{Si}$ un clínker contiene $\mathrm{K}_{2} \mathrm{O}$ y $\mathrm{Na}_{2} \mathrm{O}$ a la vez su influencia es aditiva.

\section{B I B L I O G R A F I A}

[1] TaYlor, W. C.: Journ. Res. Nat. Bur. Standards 29 (1942) S. 437-451.

[2] Eubank, W. R.: Journ. Res. Nat. Bur. Standards 44 (1950) S. 175-192.

[3] Newkirk, T. F.: Proceed. of the Third Intern Symp. on the Chem. of Cem., London 1952.

[4] Suzukawa, Y.: Zement-Kalk-Gips 9 (1956) S. 345-351, S. 390-396, S. 433-436.

[5] Thilo, E., Funk, H.: Zeitschr. anorg. allgem. Chemie 273, 1-2 (1953) S. 28-40.

[6] Niesel, K., Thormann, P.: Tonind. Z. 91 (1967) 9 S. 362-369.

[7] AselitzkaJA, R. D.: Silikattechnik 5 (1954) S. 120.

[8] DAY, D. E.: ASTM-Meeting, Juni 1965, Purdue Univ.

[9] Yamaguchr, G., u.a.: Semento Gijutsu Nenpo 17 (1963) S. 37-41.

[10] Brownmiller, L. T., Bogue, R. H.: Journ. Res. Nat. Bur. Standards 8 (1932) S. 289-307.

[11] Conwicke, J. A., Day, D. E.: Journ. Americ. Ceram. Soc. 47 (1964) 12, S. 654-655.

[12] Fletcher, K. E., Midgley, H. G., Moore, A. E.: Mag. Conc. Research 17 (1965) Nr. 53.

[13] MUssgnug, G.: Zement-Kalk-Gips 15 (1962) S. 197.

[14] KRYzhanovskajy, J. A., Mirak' Jan, V. M.: Cement (Leningrad) 1965 Sep./Okt.

[15] Wofrmann, E., Eysel, W., und HaHN, Th.: Zement-Kak-Gips 21 (1968) S. $241-251$.

[16] TrojeR, E.: Zement-Kalk-Gips 19 (1966) S. 207-215.

[17] SmolczyK, H. G.: Schriftenreihe der Zementindustrie, Heft 29 (1962), S. 31-77.

[18] KRÄmer, H., und zur Strassen, H.: Intern. Symp. on the Chem. of Cem., Wasington 1960, vol. I, S. $32-33$.

[19] KNÖFEL, D., und Spohn, E.: Zement-Kalk-Gips 22 (1969) S. 471-476. 